July 1993

ITP-SB-93-40

\title{
Scattering Matrix And Excitation Spectrum \\ OF THE HubBard Model
}

\author{
Fabian H.L.Eßler ${ }^{b}$ \\ and \\ Vladimir E. Korepin $\sharp$ \\ Institute for Theoretical Physics \\ State University of New York at Stony Brook \\ Stony Brook, NY 11794-3840
}

\begin{abstract}
We consider the one-dimensional Hubbard model at half filling. We show that both excitation spectrum and S-matrix are determined by the $\mathrm{SO}(4)$ symmetry of the model. The complete set of excitations is given by the scattering states four elementary excitations, which form the fundamental representation of $\mathrm{SO}(4)$. We evaluate the exact S-matrix, which satisfies the Yang-Baxter relation. The results for the repulsive and attractive Hubbard model are related by an interchange of spin and charge degrees of freedom.
\end{abstract}

PACS: $75.10 . \mathrm{JM}, 05.50 . \mathrm{Q}$

\footnotetext{
bE-MAIL: FABMAN@MAX.PHYSICS.SUNYSB.EDU

Address After Sept.1, 1993: Physikalisches Institut der Universität Bonn, Nussallee 12, 53115 Bonn, Germany

\#E-MAIL:KOREPIN@MAX.PHYSICS.SUNYSB.EDU
} 
Strongly correlated electronic systems are currently under intense study in relation with high- $T_{c}$ superconductivity. The two-dimensional Hubbard model is in the very center of these investigations. It is believed to share important features with its one-dimensional analog ${ }^{[1]}$. A very interesting problem is the separation of spin and charge in the Hubbard model in one $^{[2,3]}$ and two dimensions ${ }^{[4]}$. In one spatial dimension the Hubbard model can be solved exactly by means of the Bethe Ansatz ${ }^{[5]}$. The Hubbard hamiltonian is given by the following expression

$$
H=-\sum_{j=1}^{L} \sum_{\sigma=\uparrow, \downarrow}\left(c_{j, \sigma}^{\dagger} c_{j+1, \sigma}+c_{j+1, \sigma}^{\dagger} c_{j, \sigma}\right)+4 U \sum_{j=1}^{L}\left(n_{j, \uparrow}-\frac{1}{2}\right)\left(n_{j, \downarrow}-\frac{1}{2}\right)
$$

Here $c_{j, \sigma}^{\dagger}$ are canonical fermionic creation operators on the lattice, $j$ labels the sites of a chain of even length $L, \sigma$ labels the spin degrees of freedom, $U$ is the coupling constant, and $n_{j, \sigma}=c_{j, \sigma}^{\dagger} c_{j, \sigma}$ is the number operator for spin $\sigma$ on site $j$. The hamiltonian (1) is invariant under a $\mathrm{SO}(4)=\mathrm{SU}(2) \times \mathrm{SU}(2) / Z_{2}$ algebra ${ }^{[6-8]}$. The two $\mathrm{SU}(2)$ algebras are the spin $\mathrm{SU}(2)$ with generators

$$
S=\sum_{j=1}^{L} c_{j, \uparrow}^{\dagger} c_{j, \downarrow}, \quad S^{\dagger}=\sum_{j=1}^{L} c_{j, \downarrow}^{\dagger} c_{j, \uparrow}, \quad S^{z}=\sum_{j=1}^{L} \frac{1}{2}\left(n_{j, \downarrow}-n_{j, \uparrow}\right)
$$

and the $\eta$-SU(2) algebra with generators

$$
\eta=\sum_{j=1}^{L}(-1)^{j} c_{j, \uparrow} c_{j, \downarrow}, \quad \eta^{\dagger}=\sum_{j=1}^{L}(-1)^{j} c_{j, \downarrow}^{\dagger} c_{j, \uparrow}^{\dagger}, \quad \eta^{z}=\frac{1}{2} \sum_{j=1}^{L}\left(n_{j, \uparrow}+n_{j, \downarrow}-1\right) .
$$

All 6 generators commute with the hamiltonian (1). Below we first give a complete classification of the excitation spectrum of the repulsive Hubbard model by means of its SO(4) symmetry. The results are such that the spin and charge degrees of freedom completely separate from one another on the level of the elementary excitations. We then determine the exact two-particle S-matrix, which is of importance in the study of transport properties ${ }^{[9]}$. The S-matrix is $\mathrm{SO}(4)$ invariant and satisfies the Yang-Baxter relation, which implies the 2-particle reducibility of the N-body scattering matrix. We conclude with a discussion of excitation spectrum and S-matrix of the attractive Hubbard model. Detailed derivations of the results presented in this letter can be found in [10].

Let us now turn to a classification of the excitation spectrum over the repulsive half-filled ground state $(U>0)$. In the many previous works on this problem (most notably $[2,3,11])$ the correct dispersions for many excitations were obtained. However, the Lie-algebraic structure of the excitation spectrum was not fully revealed. We classify all excitations with respect to representations of the $\mathrm{SO}(4)$ symmetry algebra. The spectrum is described by the scattering states of four elementary excitations only. Two of the elementary excitations carry spin 
but no charge. This means that they transform in the spin- $\frac{1}{2}$ representation of the spin$\mathrm{SU}(2)$, and are a singlet with respect to the $\eta$-SU(2). The resulting $\mathrm{SO}(4)$-representation is denoted by $\left(\frac{1}{2}, 0\right)$. We shall call these two excitations spinons (They are similar to the two elementary excitations in the spin- $\frac{1}{2}$ Heisenberg XXX antiferromagnet at zero magnetic field $\left.{ }^{[12-14]}\right)$. Energy and momentum of these particles are expressed in terms of a spectral parameter $\lambda$. It plays the same role as the rapidity for the case of relativistic particles. Energy $\epsilon_{s}(\lambda)$ and momentum $p_{s}(\lambda)$

$$
\begin{array}{ll}
p_{s}(\lambda)=\frac{\pi}{2}-\int_{0}^{\infty} \frac{d \omega}{\omega} \frac{J_{0}(\omega) \sin (\omega \lambda)}{\cosh (\omega U)} \quad 0<p_{s}(\lambda)<\pi \\
\epsilon_{s}(\lambda)=2 \int_{0}^{\infty} \frac{d \omega}{\omega} \frac{J_{1}(\omega) \cos (\omega \lambda)}{\cosh (\omega U)} .
\end{array}
$$

Here $J_{n}(\omega)$ are Bessel functions. This dispersion relation was obtained in $[2,11]$.

The other two elementary excitations carry charge $\pm e$ but no spin. They are called holon and antiholon and form the $\left(0, \frac{1}{2}\right)$-representation of $\mathrm{SO}(4)$, i.e. they transform in the spin- $\frac{1}{2}$ representation of the $\eta$-SU(2) (3) and are a singlet with respect to the spin-SU(2)(2). The momentum of holon and antiholon differs by $\pi$ as $\eta^{\dagger}$ does not commute with the momentum operator but changes the momentum by $\pi$. The holon carries charge $+e$, where $-e$ is the charge of the electron. Energy and momentum are parametrized by the "bare" momentum $k^{h}$ of the holon, and $\sin \left(k^{h}\right)$ plays the role of the spectral parameter. Energy and momentum are given by ${ }^{[3]}$

$$
\begin{aligned}
& p_{h}\left(k^{h}\right)=\frac{\pi}{2}-k^{h}-\int_{0}^{\infty} \frac{d \omega}{\omega} \frac{J_{0}(\omega) \sin \left(\omega \sin \left(k^{h}\right)\right) e^{-\omega U}}{\cosh (\omega U)} \\
& \epsilon_{h}\left(k^{h}\right)=2 U+2 \cos \left(k^{h}\right)+2 \int_{0}^{\infty} \frac{d \omega}{\omega} \frac{J_{1}(\omega) \cos \left(\omega \sin \left(k^{h}\right)\right) e^{-\omega U}}{\cosh (\omega U)} .
\end{aligned}
$$

The antiholon can be obtained from the wave function of the holon by applying the operator $\eta^{\dagger}$. Its energy and momentum are found to be

$$
\epsilon_{a h}(k)=\epsilon_{h}(k), \quad p_{a h}(k)=-\pi+p_{h}(k)
$$

The spectrum of all excitations of the repulsive half-filled Hubbard model is given by the scattering states of the four elementary excitations listed above. The proof of this is similar to the proof of the corresponding statement in the spin- $\frac{1}{2}$ Heisenberg XXX antiferromagnet $^{[12-14]}$. The basis of the proof is a theorem of [15], which states that (i) All Bethe Ansatz states with $N_{\downarrow}<N_{\uparrow}$ below (or at) half-filling are lowest weight states of the $\operatorname{SO}(4)$ symmetry algebra, and (ii) The set of states obtained by acting with the $\mathrm{SO}(4)$ raising operators on the Bethe states is complete, i.e. it forms a basis of the electronic Hilbert space. This theorem implies that a complete set of excitations over the ground state is obtained by constructing excited states by means of the Bethe Ansatz and then act on these with 
the $\mathrm{SO}(4)$ raising operators. A complete set of Bethe Ansatz excitations can be constructed by using the zero-temperature limit of the Thermodynamic Bethe Ansatz ${ }^{[16]}$. Like in the XXX-antiferromagnet ${ }^{[12,13]}$ elementary excitations always occur in pairs. This is due to the $Z_{2}$-factor, which projects out all representations of $\mathrm{SU}(2) \times \mathrm{SU}(2)$ with half-odd integer quantum number: $S^{z}+\eta^{z}=N_{\downarrow}+\frac{L}{2}$ ( $L$ is even). Accordingly there are no one-particle excitations, and more generally no excitations with and odd number of particles (spinons or holons/antiholons) in the half-filled Hubbard model.

In order to construct the two-particle scattering matrix a complete knowledge of all excitations in the two-particle sector is necessary. In the two-particle sector there exist three kinds of excitations: Scattering states of two spinons (forming the representation $(1,0) \oplus(0,0)$ ); Scattering states of two holons (in the representation $(0,1) \oplus(0,0)$ ); and finally scattering states of one holon and one spinon (forming the $\left(\frac{1}{2}, \frac{1}{2}\right)$ representation of $\left.\mathrm{SO}(4)\right)$. Bethe states in the Hubbard model are parametrized by sets of spectral parameters $\left\{k_{j}\right\},\left\{\Lambda_{\alpha}^{n}\right\},\left\{\Lambda_{\alpha}^{\prime n}\right\}$, which are subject to the following sets of coupled algebraic equations ${ }^{[16]}$

$$
\begin{gathered}
k_{j} L=2 \pi I_{j}-\sum_{n=1}^{\infty} \sum_{\alpha=1}^{M_{n}} \theta\left(\frac{\sin k_{j}-\Lambda_{\alpha}^{n}}{n U}\right)-\sum_{n=1}^{\infty} \sum_{\alpha=1}^{M_{n}^{\prime}} \theta\left(\frac{\sin k_{j}-\Lambda_{\alpha}^{\prime n}}{n U}\right), \\
\sum_{j=1}^{N_{e}-2 M^{\prime}} \theta\left(\frac{\Lambda_{\alpha}^{n}-\sin k_{j}}{n U}\right)=2 \pi J_{\alpha}^{n}+\sum_{m=1}^{\infty} \sum_{\beta=1}^{M_{m}} \Theta_{n m}\left(\frac{\Lambda_{\alpha}^{n}-\Lambda_{\beta}^{m}}{U}\right), \\
L\left[\sin ^{-1}\left(\Lambda_{\alpha}^{\prime n}+i n U\right)+\sin ^{-1}\left(\Lambda_{\alpha}^{\prime n}-i n U\right)\right] \\
=2 \pi J_{\alpha}^{\prime n}+\sum_{j=1}^{N_{e}-2 M^{\prime}} \theta\left(\frac{\Lambda_{\alpha}^{\prime n}-\sin k_{j}}{n U}\right)+\sum_{m=1}^{\infty} \sum_{\beta=1}^{M_{m}^{\prime}} \Theta_{n m}\left(\frac{\Lambda_{\alpha}^{\prime n}-\Lambda_{\beta}^{\prime m}}{U}\right),
\end{gathered}
$$

Here $L=2 \times$ odd is the even length of the lattice, $I_{j}, J_{\alpha}^{n}$, and $J_{\alpha}^{\prime n}$ are integer or half-odd integer numbers, $N_{e}$ is the total number of electrons, $M^{\prime}=\sum_{n=1}^{\infty} M_{n}^{\prime}, \theta(x)=2 \arctan (x)$, and

$$
\theta_{n m}(x)= \begin{cases}\theta\left(\frac{x}{|n-m|}\right)+2 \theta\left(\frac{x}{|n-m|+2}\right)+\ldots+2 \theta\left(\frac{x}{n+m-2}\right)+\theta\left(\frac{x}{n+m}\right) & \text { if } n \neq m \\ 2 \theta\left(\frac{x}{2}\right)+2 \theta\left(\frac{x}{4}\right)+\ldots+2 \theta\left(\frac{x}{2 n-2}\right)+\theta\left(\frac{x}{2 n}\right) & \text { if } n=m .\end{cases}
$$

The spectral parameters $\Lambda_{\alpha}^{n}, \Lambda_{\alpha}^{\prime n}$ are the real centers of complex roots ("strings" of lenght $n$ ) of the Lieb-Wu equations (see [16]), $M_{n}$ and $M_{n}^{\prime}$ are the numbers of $\Lambda$-strings of length $n$ and $\Lambda^{\prime}$-strings of length $n$ in a specific solution of the system (7). The integer (half-odd integer) numbers in (7) have the ranges

$$
\begin{aligned}
& \left|J_{\alpha}^{n}\right| \leq \frac{1}{2}\left(N_{e}-2 M^{\prime}-\sum_{m=1}^{\infty} t_{n m} M_{m}-1\right) \\
& \left|J_{\alpha}^{\prime n}\right| \leq \frac{1}{2}\left(L-N_{e}+2 M^{\prime}-\sum_{m=1}^{\infty} t_{n m} M_{m}^{\prime}-1\right),-\frac{L-1}{2} \leq I_{j} \leq \frac{L-1}{2}
\end{aligned}
$$


where $t_{m n}=2 \min \{m, n\}-\delta_{m n}$. Each set of "integers" $\left\{I_{j}\right\},\left\{J_{\alpha}^{n}\right\},\left\{J_{\alpha}^{\prime n}\right\}$ is in one-to-one correspondence with a set of spectral parameters, which in turn unambiguously specifies one eigenstate of the hamiltonian (1). Thus the ground state and all excited states can be constructed by specifying sets of "integers" in (7) and then taking the thermodynamic limit, in which (7) reduce to coupled integral equations (see e.g. [5,2,3,11]). The half-filled ground state is obtained by taking $M_{1}=\frac{L}{2}$ and $N_{e}=L$ in (7) $\left(M_{n}=0 \forall n \geq 2, M_{n}^{\prime}=0 \forall n\right)$ and filling all vacancies for the "integers" $I_{j}$ and $J_{\alpha}^{1}$ symmetrically around zero ${ }^{[5]}$. This amounts to filling the Fermi seas of spectral parameters $k_{j}$ between $-\pi$ and $\pi$, and of $\Lambda_{\alpha}^{1}$ between $-\infty$ and $\infty$. The ground state is an $\mathrm{SO}(4)$ singlet $(0,0)$.

The scattering states of two spinons are similar to the the XXX-antiferromagnet ${ }^{[12-14]}$. The lowest weight state of the spin-triplet $\left(S=1, S^{z}=-1, \eta=0\right)$ is obtained by diminishing the number of down spins by one as compared to the ground state $M_{1}=\frac{L}{2}-1$. This enlarges the allowed range of "integers" $J_{\alpha}^{1}(10)$ by one, so that we end up with two holes in the Fermi sea of the $\Lambda_{\alpha}^{1}$. We denote the corresponding spectral parameters by $\Lambda_{1}^{h}$ and $\Lambda_{2}^{h}$. Energy and momentum of this state are given in terms of the two spinons according to $E_{S T}=\epsilon_{s}\left(\Lambda_{1}^{h}\right)+\epsilon_{s}\left(\Lambda_{2}^{h}\right)$ and $P_{S T}=p_{s}\left(\Lambda_{1}^{h}\right)+p_{s}\left(\Lambda_{2}^{h}\right)$. The lowest weight theorem cited above guarantees that not only $S^{z}=-1$ but that also $S=1$. The two other states of the triplet can be obtained by application of $S^{\dagger}$.

The $S=0, \eta=0$ spin-singlet scattering state of two spinons is constructed by choosing $M_{1}=\frac{L}{2}-2$ and $M_{2}=1$. This introduces again two holes in the $\Lambda_{\alpha}^{1}$-distribution. In addition there is one $\Lambda$-string of lenght 2 present. The allowed range of "integers" for the 2 -string (10) is 0 only. Solving the equation (7) for the spectral parameter $\Lambda^{(2)}$ we find $\Lambda^{(2)}=\frac{\Lambda_{1}^{h}+\Lambda_{2}^{h}}{2}$. Energy and momentum of the singlet are the same as for the triplet.

The scattering state of a spinon (with spin up) and a holon can be constructed from the Bethe Ansatz by choosing $N_{e}=L-1$ and $M_{1}=\frac{£}{2}-1$, which introduces one hole in the distribution of the $k_{j}$ 's and the $\Lambda_{\alpha}^{1}$ 's respectively. We denote the corresponding spectral parameters by $k^{h}$ and $\Lambda^{h}$. The spin of this excitation is $S=\frac{1}{2}=\eta, S^{z}=-\frac{1}{2}=\eta^{z}$. Accordingly it carries charge $+e$. Energy and momentum are gven by $E_{s c}=\epsilon_{s}\left(\Lambda^{h}\right)+\epsilon_{h}\left(k^{h}\right)$ and $P_{s c}=p_{s}\left(\Lambda^{h}\right)+p_{h}\left(k^{h}\right)$. The other three states of the $\mathrm{SO}(4)$ multiplet can be obtained by applying $S^{\dagger}, \eta^{\dagger}$, and both.

The scattering state of two holons with $\eta=1, \eta^{z}=-1, S=0$ (charge triplet) is constructed by making two holes in the distribution of $k_{j}$ 's $\left(N_{e}=L-2\right)$. We denote the corresponding spectral parameters by $k_{1}^{h}$ and $k_{2}^{h}$. Energy and momentum of this state are given by $E_{C T}=$ $\epsilon_{h}\left(k_{1}^{h}\right)+\epsilon_{h}\left(k_{2}^{h}\right)$ and $P_{C T}=p_{h}\left(k_{1}^{h}\right)+p_{h}\left(k_{2}^{h}\right)$. The other two states of the multiplet are obtained by applying $\eta^{\dagger}$.

The charge singlet $S=0, \eta=0=\eta^{z}$ scattering state is characterized by taking $N_{e}=$ $L, M_{1}=\frac{L}{2}-1$ and $M^{\prime 1}=1$ (insertion of a bound state of two $k^{\prime}$ s and one $\Lambda^{\prime}$ ). This introduces again two holes in the $k_{j}$ Fermi sea, and in addition there is a so-called $\Lambda^{\prime}$-string present. The spectral parameter of the $\Lambda^{\prime}$-string is determined by the system (8) to be 
$\Lambda^{\prime 1}=\frac{\sin \left(k_{1}^{h}\right)+\sin \left(k_{2}^{h}\right)}{2}$. Energy and momentum of the $\eta$-singlet are $E_{C S}=\epsilon_{a h}\left(k_{1}^{h}\right)+\epsilon_{h}\left(k_{2}^{h}\right)$ and $P_{C S}=p_{a h}\left(k_{1}^{h}\right)+p_{h}\left(k_{2}^{h}\right)$. This completes our discription of all two-particle states.

We now turn to the evaluation of the two-body scattering matrix. In Quantum Mechanics the scattering matrix can be extracted from the asymptotics of the wave-function of the scattering state ${ }^{[17]}$. The scattering of the collective excitations described above is a quantum mechanical problem. However, the ground state is highly nontrivial, as are the many-body wave functions given by the Bethe Ansatz. In [18] it was explained how to modify the rules of quantum mechanical scattering theory in order to obtain the correct scattering matrix for Bethe Ansatz solvable models ([18] deals with the derivation of Zamolodchikov's S-matrix starting from the Bethe Ansatz). The same method has been applied to the case of the XXX Heisenberg model ${ }^{[12-14]}$ (see also [19]). More precisely, the method of [18] permits the evaluation of the logarithmic derivative of the S-matrix, and thus the S-matrix up to an overall constant factor. We have fixed this factor by considering the infinite $U$ limit of holon-holon scattering. In this limit the S-matrix must reduce to the one of free fermions, which is equal to 1 . This allows us to solve the problem of fixing the constant factor in the S-matrix of the XXX-antiferromagnet: the S-matrix for that model is given by the expression (11). The complete S-matrix for the Hubbard model is $16 \times 16$ dimensional and blockdiagonal. The block describing scattering of two spinons (with spectral parameters $\Lambda_{1}^{h}$ and $\Lambda_{2}^{h}$ ) is $4 \times 4$ matrix of the form

$$
S\left(\Lambda_{1}^{h}, \Lambda_{2}^{h}\right)=-\frac{\Gamma\left(\frac{1+i \mu}{2}\right) \Gamma\left(1-\frac{i \mu}{2}\right)}{\Gamma\left(\frac{1-i \mu}{2}\right) \Gamma\left(1+\frac{i \mu}{2}\right)}\left(\frac{\mu}{\mu+i} I+\frac{i}{\mu+i} \Pi\right),
$$

where $\mu=\frac{\Lambda_{1}^{h}-\Lambda_{2}^{h}}{2 U}$ and

$$
I=\left(\begin{array}{llll}
1 & 0 & 0 & 0 \\
0 & 1 & 0 & 0 \\
0 & 0 & 1 & 0 \\
0 & 0 & 0 & 1
\end{array}\right), \Pi=\left(\begin{array}{llll}
1 & 0 & 0 & 0 \\
0 & 0 & 1 & 0 \\
0 & 1 & 0 & 0 \\
0 & 0 & 0 & 1
\end{array}\right)
$$

The physical region is defined as $\mu>0$ (only then scattering takes place). As a function of the variable $\mu$ the S-matrix (11) coincides with the S-matrix of the spin- $\frac{1}{2}$ Heisenberg antiferromagnet. This does not hold as a function of the momentum. The $4 \times 4$ block describing the scattering of two charged particles (holons/antiholons) with spectral parameters $\sin \left(k_{1}^{h}\right)$ and $\sin \left(k_{2}^{h}\right)$ is

$$
S\left(\sin \left(k_{1}^{h}\right), \sin \left(k_{2}^{h}\right)\right)=\frac{\Gamma\left(\frac{1-i \mu}{2}\right) \Gamma\left(1+\frac{i \mu}{2}\right)}{\Gamma\left(\frac{1+i \mu}{2}\right) \Gamma\left(1-\frac{i \mu}{2}\right)}\left(\frac{\mu}{\mu-i} I-\frac{i}{\mu-i} \Pi\right),
$$

where $\mu=\frac{\sin \left(k_{1}^{h}\right)-\sin \left(k_{2}^{h}\right)}{2 U}>0$. The block describing the scattering of one spinon on one holon/antiholon is purely diagonal 


$$
S\left(\Lambda^{h}, \sin \left(k^{h}\right)\right)=-i \frac{1+i \exp (\pi \mu)}{1-i \exp (\pi \mu)} I, \mu=\frac{\Lambda^{h}-\sin \left(k^{h}\right)}{2 U}>0 .
$$

The last block describes the scattering of one holon/antiholon on one spinon and is given by the same expression (14), where now $\mu=\frac{\sin \left(k^{h}\right)-\Lambda^{h}}{2 U}>0$. The complete S-matrix satisfies the Yang-Baxter equation, which implies that the many-body scattering matrix factorizes into the product of two-body S-matrices. Clearly the S-matrix reflects the SO(4) symmetry of the model. The analytical structure of the S-matrix is as follows: The allowed strip for rapidities is $\left|\operatorname{Im}\left(\Lambda^{h}\right)\right| \leq U$, and $\left|\operatorname{Im}\left(k^{h}\right)\right| \leq 2 U$. The S-matrix has poles in the complex rapidity plane, but all of them are unphysical and do not correspond to bound states.

The structure of elementary excitations in the attractive Hubbard model is as follows: there is a total of four elementary excitations, grouped into doublets of the spin and $\eta$-SU(2) respectively. The chargeless spin-carriers have the dispersion

$$
\begin{aligned}
& p_{s}(k)=\pi-k-\int_{0}^{\infty} \frac{d \omega}{\omega} \frac{J_{0}(\omega) \sin (\omega \sin (k)) e^{-\omega|U|}}{\cosh (\omega U)} \\
& \epsilon_{s}(k)=2|U|+2 \cos (k)+2 \int_{0}^{\infty} \frac{d \omega}{\omega} \frac{J_{1}(\omega) \cos (\omega \sin (k)) e^{-\omega|U|}}{\cosh (\omega U)}
\end{aligned}
$$

which up to the shift of momentum by $\frac{\pi}{2}$ is the same as for the holon in the repulsive case. The spinless charge-carriers (one particle, one hole) have the dispersions ${ }^{[20]}$

$$
\begin{aligned}
& p_{c}^{p}(\lambda)=\pi-\int_{0}^{\infty} \frac{d \omega}{\omega} \frac{J_{0}(\omega) \sin (\omega \lambda)}{\cosh (\omega U)}=\pi-p_{c}^{h}(\lambda), \\
& \epsilon_{c}^{p}(\lambda)=2 \int_{0}^{\infty} \frac{d \omega}{\omega} \frac{J_{1}(\omega) \cos (\omega \lambda)}{\cosh (\omega U)}=\epsilon_{c}^{h}(\lambda)
\end{aligned}
$$

which are (up to a constant in the momenta) the same as the spinon dispersions in the repulsive case. The S-matrix in the attractive case coincides with the one for the repulsive case as a function of the unformising parameter $\mu$. There also exists a naive argument for the relation of attractive and repulsive Hubbard model by an interchange of charge and spin degrees of freedom. It is a well known fact that attractive and repulsive Hubbard model are connected by the particle-hole transformation (for spin-up only)

$$
c_{j, \uparrow} \leftrightarrow(-1)^{j} c_{j, \uparrow}^{\dagger}
$$

under which the hamiltonian (1) transforms according to $H(U) \rightarrow H(-U)$. The transformation (17) effectively interchanges spin and charge degrees of freedom, e.g. transforms the spin-SU(2) (2) into the $\eta$-SU(2) (3) and vice versa.

In this letter we classified the excitation spectrum and the Scattering matrix of the one-dimensional Hubbard model by means of its $S O(4)$ symmetry. This emphasizes the importance of symmetries in systems of strongly correlated electrons. The S-matrix is an 
important tool in the study of transport properties ${ }^{[9]}$. The classification of elementary excitations into spinons (which carry spin but no charge) and holons/antiholons (which carry charge but no spin) gives a precise meaning to spin-and charge separation in one and twodimensional models of correlated electrons. In integrable models the S-matrix is often proportional to the R-matrix. In [21] a R-matrix for an embedding of the Hubbard hamiltonian in a commuting family of transfer matrices was derived. This R-matrix, which belongs to a fundamental model (where L-operator and R-matrix are essentially identical ${ }^{[19]}$ ), is different from our S-matrix. It would be interesting to search for a different embedding of the Hubbard hamiltonian, using a $\mathrm{SO}(4)$ invariant R-matrix proportional to our S-matrix. The L-operator for such a construction would have to be different from the R-matrix.

\section{ACKNOWLEDGEMENTS:}

It is a pleasure to thank Prof. C.N. Yang and Dr. E. Melzer for stimulating discussions. This work was supported in part by NATO 9.15.02 RG 901098 Special Panel on Chaos Order and Patterns 'Functional Integral Methods in Statistical Mechanics and Correlations'.

\section{REFERENCES}

1. P.W. Anderson, Science 235 (1987) 1196.

2. F. Woynarovich, J. Physics C16 (1983) 5293.

3. F. Woynarovich, J. Physics C15 (1982) 85.

4. G. Baskaran, Z. Zou, P.W. Anderson, Solid State Comm. 63 (1987) 973.

5. E.H. Lieb, F.Y. Wu, Phys. Rev. Lett. 20 (1968) 1445.

6. O.J. Heilmann, E.H. Lieb, Ann. New York Acad. Sci. 172 (1971) 583.

7. C.N. Yang, Phys. Rev. Lett. 63 (1989) 2144.

8. C.N. Yang and S. Zhang, Mod. Phys. Lett. B4 (1990) 759.

9. J. Carmelo, A.A. Ovchinnikov, J. Physics Cond. Mat. 3 (1991) 757.

10. F.H.L. Eßler, V.E. Korepin, SO(4) Invariant Scattering Matrix of the Hubbard Model, preprint 11. A.A. Ovchinnikov, Sov. Phys. JETP 30 (1970) 1160.

12. L.D. Faddeev, L. Takhtajan, J. Soviet Math. 24 (1984) 241.

13. L.D. Faddeev, L. Takhtajan, Phys. Lett. 85A (1981) 375.

14. L. Takhtajan, Phys. Lett. 87A (1982) 479.

15. F.H.L. Eßler, V.E. Korepin, K. Schoutens, Nucl. Phys. B372 (1992) 559, Nucl. Phys. B384 (1992) 431, Phys. Rev. Lett. 67 (1991) 3848.

16. M. Takahashi, Prog. Theor. Phys. 47 (1972) 69.

17. L.D. Landau, E.M. Lifshitz, "Quantum Mechanics", Pergamon Press 1975.

18. V.E. Korepin, Theor. Mat. Phys. 76 (1980) 165.

19. V.E. Korepin, G. Izergin and N.M. Bogoliubov, Quantum Inverse Scattering Method, Correlation Functions and Algebraic Bethe Ansatz, Cambridge University Press, 1993

20. F. Woynarovich, J. Physics C16 (1983) 6593.

21. B.S. Shastry, J. Stat. Phys. 50 (1988) 57. 